\section{About InPharm}

InPharm Association - A Young Pharmacists Group of India (www. inpharm.org), a scientific and educational society founded in 2003. For last 5 years, InPharm has contributed in different ways to the advancement of Pharmacy. Its members share interests related to Pharmacy, which they learn through active interaction. InPharm Communique and Journal of Young Pharmacists are official publications of InPharm Association.

\section{About Journal}

All contribution to J Young Pharm are reviewed by peer review process and copyediting process with the understanding that they have not been published previously and are not under consideration for publication elsewhere. Author/s is/are responsible for all statements made in their work and obtaining necessary permission to republish any previously published illustrations and/or other relevant materials. The journal's full text is available online at www.jyoungpharm.in. The journal allows free access (Open Access) to its contents and permits authors to selfarchive final accepted version of the articles on any OAl-compliant institutional / subject-based repository. The journal does not charge for submission, processing except for publication of manuscripts and for color reproduction of photographs.

\section{Article Processing Charges}

Journal of Young Pharmacists is an Open Access Journal of InPharm Association. Due to high publishing cost and other financial constraints in the year 2011, Articles clearing in peer review process or upon acceptance, Authors are required to pay a minimum of Rs. 2500/(Indian Authors) and 150/- USD (Foreign Authors) towards pre-press charges to Medknow Publications and Media Pvt. Ltd, Mumbai (this cost is for a standard length article i.e., 1-6 pages and 2 figures, articles over this limit will incur higher charges). The pre-press charges should reach within 15 days of the acceptance mail notification. Failure to pay pre-press charges will not be considered for pagination. Upon acceptance, payments can be made via http://www.journalonweb. com/jyp or by sending DD/Cheque should be in favour of "Medknow Publications and Media Pvt. Ltd." payable at Mumbai, INDIA. [w.e.f 17th December, 2011].

Final article layout: Above charges are applicable for a minimum of 1-6 pages. For more than 6 pages, add charges, of Rs. 350/extra page [Indian Authors] or 10 USD[Foreign authors]/extra page.

\section{Why do we charge?}

This journal is an Open Access, where in the content is free to read. The journal is non-commercial and currently, it is self financing and not supported by any major organizations. Cost is being absorbed only with subscriptions. Hence, a nominal cost will be levied to cover the cost of the following.

- Journal hosting systems [Medknow Publications and Media Pvt. Ltd., Mumbai]

- Manuscript submission systems [Journaloweb.com]

- In-house copyediting and linguistic support

- Pagination \& typesetting

- Inclusion in Crossref, giving a permanent DOI and enabling easy citation in other journals

- Archiving in Pubmed, where appropriate, in an internationally recognized, open access repositories

Send covering letter stating article title, manuscript number along with $\mathrm{DD} /$ cheque to

- The Editor, J Young Pharm. c/o Medknow Publications and Media Pvt. Ltd.B-9, Kanara Business Centre, Off Link Road, Ghatkopar (E), Mumbai - 400075, INDIA.

Note : InPharm Members will get $50 \%$ discount on pre-press charges.

\section{Scope of the Journal}

The journal will cover research studies/reviews related to Pharmacy subjects including some of the allied subjects. Articles with timely interest and newer research concepts will be given more preference.

\section{Editorial Policy}

Authors should prepare their manuscripts submitted to the journal exactly according to the instructions given here. Manuscripts which do not follow the format and style of the journal may be returned to the authors for revision or rejected. The journal reserves the right to make any further formal changes and language corrections necessary in a manuscript accepted for publication so that it conforms to the formatting requirements of the journal. Manuscripts and figures are not returned to the authors, not even upon rejection of the paper

\section{The Editorial Process}

A manuscript will be reviewed for possible publication with the understanding that it is being submitted to Journal of Young Pharmacists alone at that point in time and has not been published anywhere, simultaneously submitted, or already accepted for publication elsewhere. The journal expects that authors would authorize one of them to correspond with the Journal for all matters related to the manuscript. All manuscripts received are duly acknowledged. On submission, editors review all submitted manuscripts initially for suitability for formal review. Manuscripts with insufficient originality, serious scientific or technical flaws, or lack of a significant message are rejected before proceeding for formal peer-review. Manuscripts that are unlikely to be of interest to the Journal of Young Pharmacists readers are also liable to be rejected at this stage itself.

Manuscripts that are found suitable for publication in Journal of Young Pharmacists are sent to two or more expert reviewers. During submission, the contributor is requested to provide names of two or three qualified reviewers who have had experience in the subject of the submitted manuscript, but this is not mandatory. The reviewers should not be affiliated with the same institutes as the contributor/s. However, the selection of these reviewers is at the sole discretion of the editor. The journal follows a double-blind review process, wherein the reviewers and authors are unaware of each other's identity. Every manuscript is also assigned to a member of the editorial team, who based on the comments from the reviewers takes a final decision on the manuscript. The comments and suggestions (acceptance/ rejection/ amendments in manuscript) received from reviewers are conveyed to the corresponding author. If required, the author is requested to provide a point by point response to reviewers' comments and submit a revised version of the manuscript. This process is repeated till reviewers and editors are satisfied with the manuscript.

Manuscripts accepted for publication are copy edited for grammar, punctuation, print style, and format. Page proofs are sent to the corresponding author. The corresponding author is expected to return the corrected proofs within three days. It may not be possible to incorporate corrections received after that period. The whole process of submission of the manuscript to final decision and sending and receiving proofs is completed online. To achieve faster and greater dissemination of knowledge and information, the journal publishes articles online as 'Ahead of Print' immediately on acceptance.

\section{Authorship criteria}

Authorship credit should be based only on substantial contributions to each of the three components mentioned below:

- Concept and design of study or acquisition of data or analysis and interpretation of data

- Drafting the article or revising it critically for important intellectual content; and

- Final approval of the version to be published 
Participation solely in the acquisition of funding or the collection of data does not justify authorship. General supervision of the research group is not sufficient for authorship. Each contributor should have participated sufficiently in the work to take public responsibility for appropriate portions of the content of the manuscript. The order of naming the contributors should be based on the relative contribution of the contributor towards the study and writing the manuscript. Once submitted the order cannot be changed without written consent of all the contributors. The journal prescribes a maximum number of authors for manuscripts depending upon the type of manuscript, its scope and number of institutions involved (vide infra). The authors should provide a justification, if the number of authors exceeds these limits.

\section{Contribution Details}

Contributors should provide a description of contributions made by each of them towards the manuscript. Description should be divided in following categories, as applicable: concept, design, definition of intellectual content, literature search, clinical studies, experimental studies, data acquisition, data analysis, statistical analysis, manuscript preparation, manuscript editing and manuscript review. Authors' contributions will be printed along with the article. One or more author should take responsibility for the integrity of the work as a whole from inception to published article and should be designated as 'guarantor'.

\section{Conflicts of Interest/ Competing Interests}

All authors of must disclose any and all conflicts of interest they may have with publication of the manuscript or an institution or product that is mentioned in the manuscript and/or is important to the outcome of the study presented. Authors should also disclose conflict of interest with products that compete with those mentioned in their manuscript.

\section{Submission of Manuscripts}

Manuscripts must conform to the "Uniform Requirements for Manuscripts Submitted to Biomedical Journals" http://www.icmje.org/. Contributions and Manuscripts must be written in English and submitted exclusively to JYP. Manuscripts must be typewritten (double-spaced) with liberal margins and space at the top and bottom of the page. All manuscripts must be submitted on-line through the website www. journalonweb.com/jyp. First time users will have to register at this site. Registration is free but mandatory. Registered authors can keep track of their articles after logging into the site using their user name and password. Authors have to pay for submission, processing or publication of articles. If you experience any problems, please contact the editorial office by e-mail at editor [AT] jyoungpharm [DOT] in.

\section{Covering Letter}

Disclose all possible conflicts of interest (e.g., funding sources for consultancies or studies of products). A brief indication of the importance of the paper to the field of Pharmacy is helpful in gaining appropriate peer review.

\section{Copyright Form}

The contributors' / copyright transfer form (template provided below) has to be submitted in original with the signatures of all the contributors within two weeks of submission via courier, fax or email (copyright AT medknow DOT com) as a scanned image. Print ready hard copies of the images (one set) or digital images should be sent to the journal office at the time of submitting revised manuscript. High resolution images (up to $5 \mathrm{MB}$ each) can be sent by email on images AT medknow DOT com).

The hard copies of the Contributors' form / copyright transfer form may be sent to the following addresses or submitted online from the authors' area on www.journalonweb.com/jyp

\section{Preparation of Manuscript}

Your Manuscript should be typed, double-spaced on standard-sized - paper (8.5" x 11") with 1" margins on all sides. You should use $12 \mathrm{pt}$ Times New Roman font. Authors should take care over the fonts which are used in the document, including fonts within graphics. Fonts should be restricted to Times New Roman, Symbol and Zapf Dingbats.

Title : Should be in Title Case; The first character in each word in the title have to be capitalized.

A research paper typically should include in the following order

- Abstract

- Keywords

- Introduction

- Materials and Methods

- Results

- Discussion

- Conclusion

- Acknowledgements (If any)

- References

- Tables and/or Figures

- Appendixes (if necessary)

- Abbreviations (if necessary)

\section{Abstract - Limit of 250 Words}

A brief summary of the research. The abstract should include a brief introduction, a description of the hypothesis tested, the approach used to test the hypothesis, the results seen and the conclusions of the work.

\section{Key words}

Please, write no more than six keywords. Write specific keywords. They should be written left aligned, arranged alphabetically in 12pt Times Roman, and the line must begin with the words Keywords boldfaced. A 12pt space should separate the keywords from the affiliations.

\section{Introduction}

Description of the research area, pertinent background information, and the hypotheses tested in the study should be included under this section. The introduction should provide sufficient background information such that a scientifically literate reader can understand and appreciate the experiments to be described. The introduction MUST include in-text citations including references to pertinent reviews and primary scientific literature. The specific aims of the project should be identified along with a rationale for the specific experiments and other work performed.

\section{Materials and Methods}

Materials and/or subjects utilized in the study as well as the procedures undertaken to complete the work. The methods should be described in sufficient detail such that they could be repeated by a competent researcher. Please include the company sources for all uncommon reagents (kits, drugs, etc). Illustrations and/or tables may be helpful in describing complex equipment or elaborate procedures. The statistical tool used to analyze the data should be mentioned. All procedures involving experimental animals or human subjects must accompany with statement on necessary ethical approval from appropriate ethics committee.

\section{Results}

Data acquired from the research with appropriate statistical analysis described in the methods section should be included in this section. The results section should describe the rational for each experiment, the results obtained and its significance. Results should be organized into figures and tables with descriptive captions. The captions, although brief, should tell the reader the method used, explain any abbreviations included in the figure, and should end with a statement as to the conclusion of the figure. Qualitative as well as quantitative results should be included if applicable.

\section{Discussion/Conclusion}

This section should relate the results section to current understanding 
of the scientific problems being investigated in the field. Description of relevant references to other work/s in the field should be included here. This section also allows you to discuss the significance of your results - i.e. does the data support the hypotheses you set out to test? This section should end with new answers/questions that arise as a result of your work.

\section{Tables and Figures}

Tables

Each table must start on a separate sheet. They should be numbered with Roman numerals according to their sequence in the text, and have a short self-explanatory heading. Use SI units. Tables should include vertical rules, but horizontal rules should separate column headings from the content. Authors should keep in mind the page layout of the journal when designing tables. Tables that fit onto one printed page are preferred. Detailed explanations of symbols, units, and abbreviations should follow below the table.

\section{Illustrations}

Figures for final production should be submitted as electronic files and hard copy so that the editorial office can ensure that the output of electronic files matches the hardcopy. Please pay particular attention to the guidelines below. The editorial office cannot undertake preparation of manuscripts and illustrations not conforming to journal style. Manuscripts of insufficient quality will be returned immediately without refereeing. A high standard of illustration (both line and photo) is an editorial priority. All illustrations should be prepared for printing to fit $80 \times 240 \mathrm{~mm}$ (column width) or $169 \mathrm{~mm}$ by up to $240 \mathrm{~mm}$ (full page) size. It is preferred that the full-page length is not used and that authors keep in mind that the caption will be placed underneath the figure. In the event that full-page length is necessary for plates, captions will have to appear on adjacent pages. Figure(s) must be numbered consecutively in the text. Compound figures with more than one micrograph or photo should be referred by a single figure reference (e.g. Figure 1), and individual parts should be labeled with capitalized letters in the lower left-hand corner. Lettering should be of a sans-serif type (i.e. fonts without serifs such as Arial) with a minimum published size of $4.2 \mathrm{~mm}$ (12 pt). Descriptive labeling in the figures should be clearly readable, and all lettering should have a minimum published size of $6 \mathrm{pt}(2.1 \mathrm{~mm})$ for labeling items on photographs or in line art is recommended and a maximum size of $10 \mathrm{pt}$ is suggested. Use a scale bar to indicate magnifications and place in the lower right corner if possible. Computer prepared photographic images must be at a minimum of $350 \mathrm{dpi}$ at the final publication size. Lower resolution will result in pixilation and poor quality images. These should be submitted as JPEG, TIFF or PPT files, but encapsulated postscript (EPS) format is also acceptable.

Computer drawn figures are accepted provided they are of high quality. Please note that graphs produced by many statistic packages are rarely adequate. In particular, letter quality on axes and captions are often poor. Such figures should be exported into an accepted graphics package and lettering rendered using a text function. Authors should note that .dot, .bmp, and .pat fills should be avoided. Do not use postscript fill patterns as these are often based on bit map patterns that result in screening patterns during final reproduction. When filling illustrations, use fills such as lines, tints or solids. Line width minimum is $0.25 \mathrm{pt}(0.09 \mathrm{~mm})$. Also avoid the use of bitmap scans to render text and detail. Text should be saved as text at a minimum text size of $6 \mathrm{pt}$ (2.1 mm). Please submit line art as Corel Draw, Adobe Illustrator, or EPS files. These must be at a minimum resolution of $800 \mathrm{DPI}$ at publication size. High resolution may be necessary where fine line detail is present. For graphs Excel graphs are also acceptable. Note that vertical axes must all be at the same scale especially where the paper compares between them. Otherwise they should be produced as separate figures. Avoid 3D plots when presenting 2D data.

Table and Figure captions

Figure and table captions should be included at the end of the manuscript. Figure captions/legends should include a statement at the end of each caption/legends about reproduction size (e.g. at full page width, at column width). They should be double spaced and typed in the journal format. Explanations should be brief and authors should keep in mind that captions/legends will be placed below figures.

\section{Acknowledgements - Limit of 100 Words}

This is a brief section crediting the people who have helped make your manuscript possible and who aided you in your work but are not part of the authorship. Please mention all applicable grants and other funding that supported your work.

\section{Review Articles}

It is expected that these articles would be written by individuals who have done substantial work on the subject or are considered experts in the field. A short summary of the work done by the contributor(s) in the field of review should accompany the manuscript.

The prescribed word count is up to 3000 words excluding tables, references and abstract. The manuscript may have about 90 references. The manuscript should have an unstructured Abstract (250 words) representing an accurate summary of the article. The section titles would depend upon the topic reviewed. Authors submitting review article should include a section describing the methods used for locating, selecting, extracting, and synthesizing data. These methods should also be summarized in the abstract.

The journal expects the contributors to give post-publication updates on the subject of review. The update should be brief, covering the advances in the field after the publication of the article and should be sent as a letter to editor, as and when major development occurs in the field.

\section{Page layout \& styles}

Page size Letter Portrait $81 / 2 \times 11$

Margins All Margins, $1 \mathrm{~cm}$

Page number Numbered at bottom right

Footer/Headers None

Title 14 pt Times New Roman, bold, centered.

Author and co-authors 12 pt Times New Roman centered, boldauthor and all co-authors names in one line. The corresponding author should include an asterisk*.

Authors address $\quad 12$ pt Times New roman centered - giving each authors' affiliation (i.e. Department/ Organization/Address/Place/Country/ email). Followed by single line spacing. Author for Correspondence: 10pt Times New roman centered - giving a valid e-mail of the corresponding (main) author is a must. It should be indicated as* followed by two line spacing.

Abstract 12 pt Times New roman, full justification Normal - maximum 250 words

Text

12 pt Times New roman, full justification 1.5 line spacing between paragraphs. No indentation

Heading Major headings (ABSTRACT, KEYWORDS, INTRODUCTION, MATERIALS AND METHODS, RESULTS AND DISCUSSION, ACKNOWLEDGEMENTS, REFERENCES) in upper case left-justified, 12 pt bold, Intermediate headings should be in italics, sentence case, left justified, $12 \mathrm{pt}$ 
Tables

To be incorporated at the end of Manuscript

Correct

"Table 1: Serum enzyme levels........"

Incorrect

"Table No. 1: Serum enzyme levels........."

Figures/Graphs Figures may be embedded in your word document but they should be created with a program that allows you to save them as gif, jpg or tiff format.

For any figures or other materials directly extracted from previously published materials, you must have written permission from the publisher of that material for reprint use. A copy of that permission release must be submitted with your article.

It is the individual author's responsibility to attain this permission.

To be incorporated at the end of the manuscript with proper labeling

Correct

"Figure 1 : Serum enzyme levels........"

Incorrect

"Figure No. 1 : Serum enzyme levels........"

Graphs To be included from excel, it should be editable. Non - editable graphs will not be accepted.

All text should bully justified. Please put all primary section titles in ER CASE letters and subheading in both Upper and Lower Case letters. Do not number your titles (for example, 1.0 Introduction; 2.0 Background). Do not use the t key to indent blocks of text such as paragraphs of quotes or lists because the page layprogram overrides your left margin with its own, and tabs end up in mid-sentence.

\section{References}

Reference List: Author/Authors < References should be numbered consecutively in the order i which they are first mentioned in the text (not in alphabetic order). nscript with square bracket after the punctuation marks. References cited only in tables or figure legends should be numbered in accordance with the sequence established by the first identification in the text of the particular table or figure. Use the style of the examples below, which are based on the formats used by the NLM in Index Medicus. The titles of journals should be abbreviated according to the style used in Index Medicus. Use complete name of the journal for non-indexed journals. Avoid using abstracts as references. Information from manuscripts submitted but not accepted should be cited in the text as "unpublished observations" with written permission from the source. Avoid citing a "personal communication" unless it provides essential information not available from a public source, in which case the name of the person and date of communication should be cited in parentheses in the text. The commonly cited types of references are shown here, for other types of references such as newspaper items please refer to ICMJE Guidelines (http://www.icmje.org or http://www.nlm.nih.gov/bsd/ uniform_requirements.html).

Download a PowerPoint presentation on common reference styles and using the reference checking facility on the manuscript submission site.

\section{In-text citation examples}

\section{Correct/Acceptable Format}

Natural products have proven to be a great source of new biologically active compounds. Thus, in an effort to discover new lead anti-malarial compounds, several research group screen plant extracts to detect secondary metabolites with relevant biological activities that could served as templates for the development of new drugs. Flavonoids have been isolated and characterized from many medicinal plants used in malaria endemic areas. ${ }^{[10]}$ However, controversial data have been obtained regarding their antiplasmodial activity, probably because of their structural diversity. ${ }^{[11-13]}$ More recently, several flavonoids have been isolated from Artemisia afra ${ }^{[14,16]}$ and Artemisia indica, ${ }^{[17-20]}$ two plants related to Artemisia annua, the famous traditional Chinese medicinal plant from which artemisinin is isolated.

\section{Incorrect/Not accepted}

Natural products have proven to be a great source of new biologically active compounds. Thus, in an effort to discover new lead anti-malarial compounds, several research group screen plant extracts to detect secondary metabolites with relevant biological activities that could served as templates for the development of new drugs. Flavonoids have been isolated and characterized from many medicinal plants used in malaria endemic areas. ${ }^{[10]}$. However, controversial data have been obtained regarding their antiplasmodial activity, probably because of their structural diversity $(11,12,13)$. More recently, several flavonoids have been isolated from Artemisia afra $^{14}$ and Artemisia indica ${ }^{(15)}$, two plants related to Artemisia annua, the famous traditional Chinese medicinal plant from which artemisinin is isolated.

\section{JOURNAL REFERENCES [Vancouver Style]}

\section{Single/Multiple Authors}

Halpern SD, Ubel PA, Caplan AL. Solid-organ transplantation in HIV-infected patients. N Engl J Med. 2002 Jul 25;347(4): 284-7.

\section{More than six authors}

Rose ME, Huerbin MB, Melick J, Marion DW, Palmer AM, Schiding JK, et al. Regulation of interstitial excitatory amino acid concentrations after cortical contusion injury. Brain Res. 2002; 935(1-2): 40-6.

\section{Organization as Author}

Diabetes Prevention Program Research Group. Hypertension, insulin, and proinsulin in participants with impaired glucose tolerance. Hypertension. 2002; 40(5): 679-86.

4. Unknown Author

21 st century heart solution may have a sting in the tail. BMJ. 2002; 325(7357): 184-5.

5. Journal article on the Internet

Abood S. Quality improvement initiative in nursing homes: the ANA acts in an advisory role. Am J Nurs [serial on the Internet]. 2002 Jun [cited 2002 Aug 12]; 102(6): [about 3 p.]. Available from: http://www.nursingworld.org/AJN/2002/june/Wawatch.htm Note: Plant/Micro organisms, in-vivo, in-vitro should be in italics.

6. Personal author(s)

Murray PR, Rosenthal KS, Kobayashi GS, Pfaller MA. Medical microbiology. 4th ed. St. Louis: Mosby; 2002.

7. Editor(s), compiler(s) as author

Gilstrap LC 3rd, Cunningham FG, VanDorsten JP, editors. Operative obstetrics. 2nd ed. New York: McGraw-Hill; 2002.

8. Author(s) and editor(s)

Breedlove GK, Schorfheide AM. Adolescent pregnancy. 2nd ed. Wieczorek RR, editor. White Plains (NY): March of Dimes Education Services; 2001.

9 Organization(s) as author Royal Adelaide Hospital; University of Adelaide, Department of Clinical Nursing. Compendium of nursing research and practice development, 1999-2000. Adelaide (Australia): Adelaide University; 2001.

\section{Chapter in a book}

Meltzer PS, Kallioniemi A, Trent JM. Chromosome alterations in human solid tumors. In: Vogelstein B, Kinzler KW, editors. The genetic basis of human cancer. New York: McGraw-Hill; 2002. p. 93-113. 


\section{Conference proceedings}

Harnden P, Joffe JK, Jones WG, editors. Germ cell tumours V. Proceedings of the 5th Germ Cell Tumour Conference; 2001 Sep 13-15; Leeds, UK. New York: Springer; 2002.

\section{Thesis}

N. Khoshakhlagh. The compositions of volatile fractions of Peganum harmala seeds and its smoke. Pharm. D. Thesis, Faculty of Pharmacy, Tehran University of Medical Sciences, Tehran, Iran. (2002).

\section{WEBSITES}

Website information

Cancer-Pain.org [homepage on the Internet]. New York: Association of Cancer Online Resources, Inc.; c2000-01 [updated 2002 May 16; cited 2002 Jul 9]. Available from: http://www.cancer-pain.org/.

\section{Acknowledgements}

All messages and reviews sent electronically will be acknowledged electronically upon receipt

\section{Submission of manuscript}

Manuscripts always be submitted only by Web based Manuscript Submission Systems[WBMS] PREFERABLY

Visit http://www.journalonweb.com/jyp

Note : Do not send hard copies/CDs, until you receive e-mail request from Editorial office.

A timely submission, however, is not a guarantee that your work will be accepted for forthcoming publication. All submissions are peer reviewed by the editorial board and a select group of reviewers. Please make sure that all guidelines are followed carefully. All the accepted articles will be queued for publication and will appear in the futures issues based on the priorities set by the editorial board.

\section{Important e-mails}

$J$ Young Pharm

Queries : editor@jyoungpharm.in

(Queries will be replied in two working days)

Other correspondence : editor @ jyoungpharm.in

\section{HARD COPY SUBMISSION}

Hard Copy of Manuscripts are not being accepted, Submissions should be made by Web Based Manuscript Submission Systems ONLY Visit : www.jyoungpharm.in

Check List before Submitting Manuscript

- Covering letter

- Copyright Forms (Scanned)

- Manuscript
- Illustrations (if any)

Book Reviews

Send One hard copy of your book, to the following address;

The Editor (Book Reviews)

Dr. Mueen Ahmed KK

Secretary, InPharm Association, H.Q.: Al-Ameen College of Pharmacy,

Near Lalbagh Main Gate,

Hosur Road, Bangalore 560027 , India.

\section{AUTHOR CHECKLIST FOR SENDING PROOFS TO EDITORIAL OFFICE}

In order to maintain quality and consistency in InPharm Publications, we ask you to perform the following checklist prior to submitting your final proof for publication:

- Include the original, hard copy of Author's Transfer of Copyright signed by each author

- Thoroughly check the reference style as mentioned above.

- Thoroughly check the article for correct grammar, in particular: spelling of names, affiliations, any symbols, equations, etc.

- $\quad$ Provide laser printed hard copies of all figures and graphics in black and white or colour (If any)

- Submit a proof corrected with RED INK ONLY or as directed by the editor handling your manuscript.

Send the Corrected Proof (scanned), Copyright Transfer Form, Subscriptions (If any) with covering letter in a single envelope to the following address

Note : e-mails with the forcibly/influential consideration, multiple queries on status, vicious language, offensive writings to Editor/Associate Editors will lead to rejection of the Manuscript.

\section{Status of Manuscript}

The Editorial team will inform the status of submitted manuscript every 15th day of month directly to the corresponding author's inbox.

Dr. Mueen Ahmed K K: editor @ jyoungpharm.in

URL:

J Young Pharm : www.jyoungpharm.in

InPharm Association : www.inpharm.org

Online Submissions : http://www.journalonweb.com/jyp 


\section{Contributors' form}

(to be modified as applicable and one signed copy attached with the manuscript)

Manuscript Title:

I/we certify that I/we have participated sufficiently in contributing to the intellectual content, concept and design of this work or the analysis and interpretation of the data (when applicable), as well as writing of the manuscript, to take public responsibility for it and have agreed to have my/ our name listed as a contributor.

I/we believe that the manuscript represents valid work. Neither this manuscript nor one with substantially similar content under my/our authorship has been published or is being considered for publication elsewhere, except as described in the covering letter. I/we certify that all the data collected during the study is presented in this manuscript and no data from the study has been or will be published separately. I/we attest that, if requested by the editors, I/we will provide the data/information or will cooperate fully in obtaining and providing the data/information on which the manuscript is based, for examination by the editors or their assignees. Financial interests, direct or indirect, that exist or may be perceived to exist for individual contributors in connection with the content of this paper have been disclosed in the cover letter. Sources of outside support of the project are named in the covering letter.

I/We hereby transfer(s), assign(s), or otherwise convey(s) all copyright ownership, including any and all rights incidental thereto, exclusively to the Journal of Young Pharmacists, in the event that such work is published by the Journal of Young Pharmacists. The Journal of Young Pharmacists shall own the work, including

1. copyright;

2. the right to grant permission to republish the article in whole or in part, with or without fee;

3. the right to produce preprints or reprints and translate into languages other than English for sale or free distribution; and

4. the right to republish the work in a collection of articles in any other mechanical or electronic format.

We give the rights to the corresponding author to make necessary changes as per the request of the journal, do the rest of the correspondence on our behalf and he/she will act as the guarantor for the manuscript on our behalf.

All persons who have made substantial contributions to the work reported in the manuscript, but who are not contributors, are named in the Acknowledgment and have given me/us their written permission to be named. If I/we do not include an Acknowledgment that means I/we have not received substantial contributions from non-contributors and no contributor has been omitted.

\section{Name}

2

3

\section{4}

5

6
Signature

Date signed

(up to 4 contributors for case report/ images/ review)

(up to 6 contributors for original studies) 